\title{
Intermolecular Packing Effects on Singlet Fission in Oligorylene Dimers
}

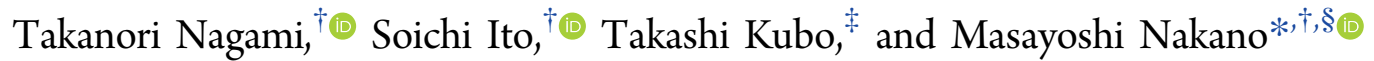 \\ ${ }^{\dagger}$ Department of Materials Engineering Science, Graduate School of Engineering Science and ${ }^{\S}$ Center for Spintronics Research \\ Network (CSRN), Graduate School of Engineering Science, Osaka University, Toyonaka, Osaka 560-8531, Japan \\ ${ }^{\ddagger}$ Department of Chemistry, Graduate School of Science, Osaka University, Toyonaka, Osaka 560-0043, Japan
}

Supporting Information

\begin{abstract}
Using the density functional theory method, the crystalline packing effect on the singlet fission (SF) rate of oligorylenes, some of which are found to exhibit SF in crystal forms, is revealed by evaluating the effective electronic coupling $\left(\left|V_{\text {eff }}\right|\right)$, the square of which is proportional to the SF rate. The $\left|V_{\text {eff }}\right|$ values for terrylene and quaterrylene dimer models are investigated for a variety of slip-stacked forms. It is found that these values show similar dependences on the intermolecular packing as a function of lateral and longitudinal displacements of monomer frameworks, and that they are maximized in several configurations of one monomer slipped from another along the longitudinal axis. The present estimation method of the SF rate is also found to qualitatively explain the experimental SF rate difference between terrylene derivatives with different packing forms. Furthermore, by analyzing the effect of electronic couplings on the adiabatic electronic states related to SF, we predict several favorable molecular packings leading to a fast SF with a high triplet yield.
\end{abstract}

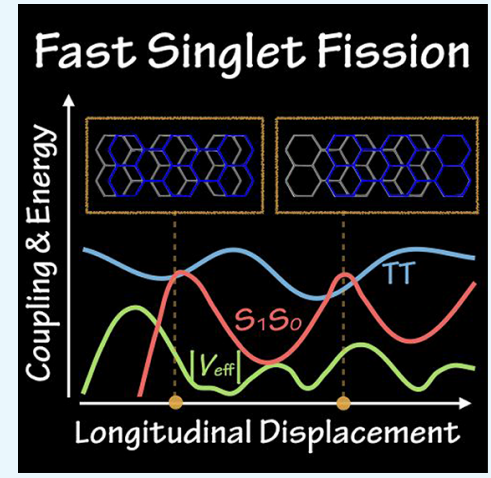

\section{INTRODUCTION}

Singlet fission (SF) is a photochemical process, which mostly occurs in organic molecular aggregates. In this process, a singlet exciton on an excited molecule interacts with another molecule in the ground state and then splits into two triplet excitons distributed over both molecules. ${ }^{1,2}$ This intriguing phenomenon has attracted much attention in the broad field of science and engineering because SF is expected to significantly improve the photoelectric conversion efficiency of organic solar cells, ${ }^{3}$ and drastically change the nonlinear optical properties. ${ }^{4,5}$ So far, a lot of experimental ${ }^{4-10}$ and theoretical ${ }^{1,2,11-14}$ studies have been conducted to reveal the mechanism as well as to design efficient SF molecular systems. ${ }^{15-18}$

To establish a guideline for screening materials that exhibit efficient SF, the energy level matching conditions for a monomer was proposed by Smith and Michl. ${ }^{1,2}$ This guideline states that molecules, which have the singlet excitation energy slightly higher than or nearly equal to twice the triplet excitation energy $\left(E\left(\mathrm{~S}_{1}\right)>2 E\left(\mathrm{~T}_{1}\right)\right.$ or $\left.E\left(\mathrm{~S}_{1}\right) \approx 2 E\left(\mathrm{~T}_{1}\right)\right)$, are energetically favorable for SF [condition (i)]. Here, $E\left(S_{1}\right)$ and $2 E\left(T_{1}\right)$ approximately represent the energies of singlet exciton $\left(\mathrm{S}_{1} \mathrm{~S}_{0}\right)$ and double-triplet exciton $\left(\mathrm{T}_{1} \mathrm{~T}_{1}\right)$ states of the dimer, respectively. In addition to condition (i), a second condition (ii) $2 E\left(\mathrm{~T}_{1}\right)<E\left(\mathrm{~T}_{2}\right)$ is required to suppress the triplet-triplet recombination of the split triplet pair. From this fundamental guideline, large alternant hydrocarbons and biradicaloids are proposed to be promising molecular systems for efficient SF. ${ }^{18}$ This guideline based on the energy level matching conditions has been refined by introducing the multiple diradical character, ${ }^{19}$ that is, diradical $\left(y_{0}\right)$ and tetraradical $\left(y_{1}\right)$ characters $\left(0 \leq y_{0}\right.$ and $\left.y_{1} \leq 1.0\right)$ : molecules which have a relatively small diradical character $\left(\sim 0.1<y_{0}<\sim 0.4\right)$ and smaller tetraradical character $\left(y_{1} / y_{0}<\sim 0.2\right)$ are candidates for efficient SF materials. ${ }^{15,16,20}$ This diradical character-based guideline can select biradicaloids suitable for efficient SF candidates and can sort out some exceptions of alternant hydrocarbons such as phenacenes, which are nearly closed-shell and do not exhibit SF. ${ }^{16}$ As a result, the feasible region for efficient SF materials is shown on the $y_{0}-y_{1}$ map. ${ }^{15}$

Following this diradical character-based design principle, oligorylenes, including perylene, terrylene, quaterrylene, and pentaterrylene, have been investigated in terms of the theoretical correlation between the diradical character and relevant excitation energies calculated using the time-dependent tuned long-range corrected density functional theory (DFT) Becke, Lee, Yang, and Parr (TD-tuned-LC-BLYP) method. ${ }^{20}$ As a result, terrylene and quaterrylene have been found to lie in the efficient SF region on the $y_{0}-y_{1}$ map and satisfy the energy level matching conditions (i) and (ii). Recently, two terrylene derivatives, which have different stacking structures in crystalline thin films, have been synthesized by Eaton et al., ${ }^{21}$ and according to our theoretical prediction, ${ }^{20}$ they have been confirmed to undergo SF by the transient absorption experiment. In addition, these derivatives have been revealed to have different triplet formation timescales and different triplet yields depending on their crystalline thin films. It has

Received: May 24, 2017

Accepted: August 14, 2017

Published: August 29, 2017 
been implied that the origin of the different SF behaviors lies in the different intermolecular configurations in crystal packing, as inferred in other theoretical and experimental studies on the relationship between SF behaviors and intermolecular packing $^{22-26}$ or intramolecular packing. ${ }^{27-30}$ From those results, stacking displacements of chromophore frameworks as well as the variation in the interchromophore distances ${ }^{31,32}$ are expected to significantly affect the SF efficiency. Thus, the clarification of the intermolecular packing effects on the SF efficiency in molecular aggregates as well as the quest for optimal intermolecular packing forms for SF are indispensable for realizing efficient SF materials.

In this study, we investigate the intermolecular packing effects on the SF of terrylene and quaterrylene because they are predicted to be good candidates for the SF monomer as mentioned above, but their electronic structures related to SF are still poorly understood in the condensed phase. We consider the dimer models composed of these compounds and clarify the influence of dimer packing forms on the SF performances by evaluating the effective electronic couplings related to the SF rate. In addition, the effective energetics for SF obtained from the related electronic couplings are investigated in various slip-stacked terrylene or quaterrylene dimer configurations, including the real crystal packing geometries. From these calculation results, we propose several favorable intermolecular packing forms for further improving the SF performances in oligorylenes as well as in related planar $\pi$-conjugated polycyclic hydrocarbons.

\section{METHODS AND MODEL}

2.1. Computational Methods. From Fermi's golden rule combined with the quasi-degenerate perturbation theory, ${ }^{33}$ the $S F$ rate from $S_{1} S_{0}$ to $T_{1} T_{1}$ is predicted to be proportional to the square of the effective electronic coupling for the SF rate $V_{\text {eff }}, 2,29,33$

$$
V_{\text {eff }}=\sqrt{\frac{3}{2}} \frac{\left\langle l_{\mathrm{A}}|F| h_{\mathrm{B}}\right\rangle\left\langle l_{\mathrm{A}}|F| l_{\mathrm{B}}\right\rangle-\left\langle h_{\mathrm{A}}|F| h_{\mathrm{B}}\right\rangle\left\langle h_{\mathrm{A}}|F| l_{\mathrm{B}}\right\rangle}{\Delta E_{\mathrm{CT}}}
$$

where

$$
\frac{1}{\Delta E_{\mathrm{CT}}}=\frac{1}{2}\left(\frac{1}{E_{\mathrm{S}_{1} \mathrm{~S}_{0}}-E_{\mathrm{CT}}}+\frac{1}{E_{\mathrm{T}_{1} \mathrm{~T}_{1}}-E_{\mathrm{CT}}}\right)
$$

$\mathrm{A}$ and $\mathrm{B}$ are indices of the molecule in the dimer subset, and $h_{i}$ and $l_{j}$ denote the highest occupied molecular orbital (HOMO) of molecule $i$ and the lowest unoccupied molecular orbital (LUMO) of molecule $j$, respectively. $E_{\mathrm{S}_{1} \mathrm{~S}_{0}}, E_{\mathrm{T}_{1} \mathrm{~T}_{1}}$, and $E_{\mathrm{CT}}$ represent the energy levels of $S_{1} S_{0}, T_{1} T_{1}$, and charge-transfer (CT) state. By the change of intermolecular packing in the dimer subset, $V_{\text {eff }}$ is thought to vary due to the change in the overlaps of the concerned molecular orbitals.

In addition to the SF rate, intermolecular packing is predicted to affect the energetics of SF. ${ }^{13,34,35}$ We investigate the effect of the electronic couplings on the nonperturbed energy levels of the initial $\left(S_{1} S_{0}\right)$ and final $\left(T_{1} T_{1}\right)$ states by applying the quasi-degenerate perturbation theory. ${ }^{33}$ By this treatment, one can consider effective SF energetics through the relative relationship of diagonal elements of the effective Hamiltonian $H_{\text {eff }}$, which is the $3 \times 3$ matrix expressed as

$$
H_{\text {eff }}=\left(\begin{array}{ccc}
E_{\mathrm{T}_{1} \mathrm{~T}_{1}}+\Delta E_{\mathrm{T}_{1} \mathrm{~T}_{1}} & & \\
V_{\text {eff }} & E_{\mathrm{S}_{1} \mathrm{~S}_{0}}+\Delta E_{\mathrm{S}_{1} \mathrm{~S}_{0}} & \\
-V_{\text {eff }} & J_{\text {eff }} & E_{\mathrm{S}_{0} \mathrm{~S}_{1}}+\Delta E_{\mathrm{S}_{1} \mathrm{~S}_{0}}
\end{array}\right)
$$

Here, $V_{\text {eff }}$ is defined in eq $1 \mathrm{a}$, and other quantities in eq $2 \mathrm{a}$ are given by

$$
\begin{aligned}
\Delta E_{\mathrm{T}_{1} \mathrm{~T}_{1}} & =\frac{\frac{3}{2}\left(\left\langle l_{\mathrm{A}}|F| h_{\mathrm{B}}\right\rangle^{2}+\left\langle h_{\mathrm{A}}|F| l_{\mathrm{B}}\right\rangle^{2}\right)}{E_{\mathrm{T}_{1} \mathrm{~T}_{1}}-E_{\mathrm{CT}}} \\
\Delta E_{\mathrm{S}_{1} \mathrm{~S}_{0}} & =\frac{\left\langle h_{\mathrm{A}}|F| h_{\mathrm{B}}\right\rangle^{2}+\left\langle l_{\mathrm{A}}|F| l_{\mathrm{B}}\right\rangle^{2}}{E_{\mathrm{S}_{1} \mathrm{~S}_{0}}-E_{\mathrm{CT}}}
\end{aligned}
$$

and

$$
J_{\text {eff }}=J_{\text {Coul }}+J_{\text {CT }}=J_{\text {Coul }}-\frac{2\left\langle h_{\mathrm{A}}|F| h_{\mathrm{B}}\right\rangle\left\langle l_{\mathrm{A}}|F| l_{\mathrm{B}}\right\rangle}{E_{\mathrm{S}_{1} \mathrm{~S}_{0}}-E_{\mathrm{CT}}}
$$

where $J_{\text {Coul }}$ is the Coulomb coupling. The details of derivation for eqs $1 \mathrm{a}, 1 \mathrm{~b}$, and $2 \mathrm{a}-2 \mathrm{~d}$ are summarized in the Supporting Information.

2.2. Model. We consider two types of oligorylenes, terrylene and quaterrylene (Figure 1), and construct slip-stack

(a)

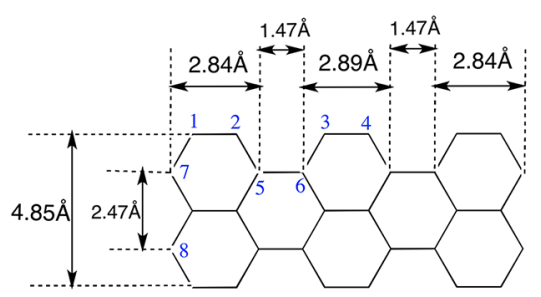

(b)

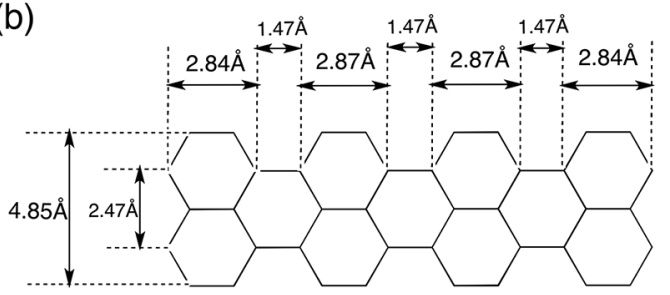

Figure 1. Structures of terrylene (a) and quaterrylene (b). The distances between carbon atoms in the optimized monomers are also shown here. Only single $\mathrm{C}-\mathrm{C}$ bonds are shown here for simplicity. The blue-colored numbers on carbon atoms indicate atom numbers.

dimer models of them (Figure 2), where $x$ and $y$ axes indicate the longitudinal and lateral directions, respectively, and $z$ axis represents the stacking direction. The center of the red-colored molecule (lower molecule of the dimer) is located at the origin of the coordinate axis. The values of $z$, interplanar distances, are fixed at $3.31 \AA$ in the terrylene dimer model and at $3.41 \AA$ in the quaterrylene dimer model; both of them are extracted from their real crystal structures. ${ }^{21,36}$ Note here that for comprehension of the primary correlation between the SF efficiency and intermolecular packing, we focus only on the dimer subset and ignore the effect of singlet exciton delocalization and polarizations of external molecules, which are not predicted to much affect the relative variation of electronic coupling. The geometries of the terrylene and quaterrylene monomers were optimized using DFT with the B3LYP functional and the 6- 


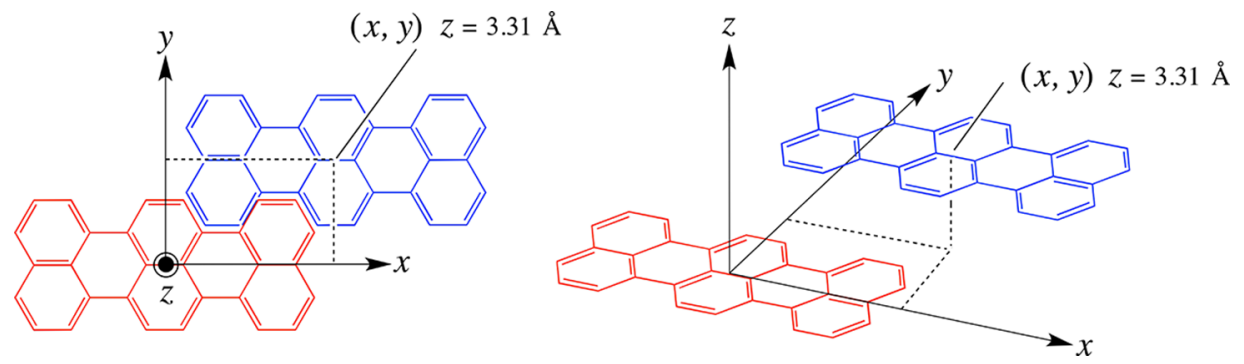

Figure 2. Cofacial terrylene dimer model and the coordinate axes. For the quaterrylene dimer model, the coordinate axis definition is the same except for $z=3.41 \AA$.

(a)

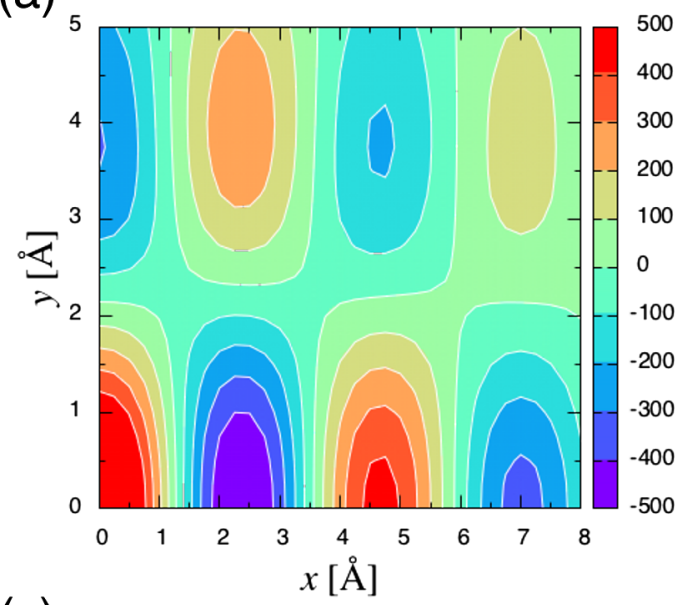

(c)

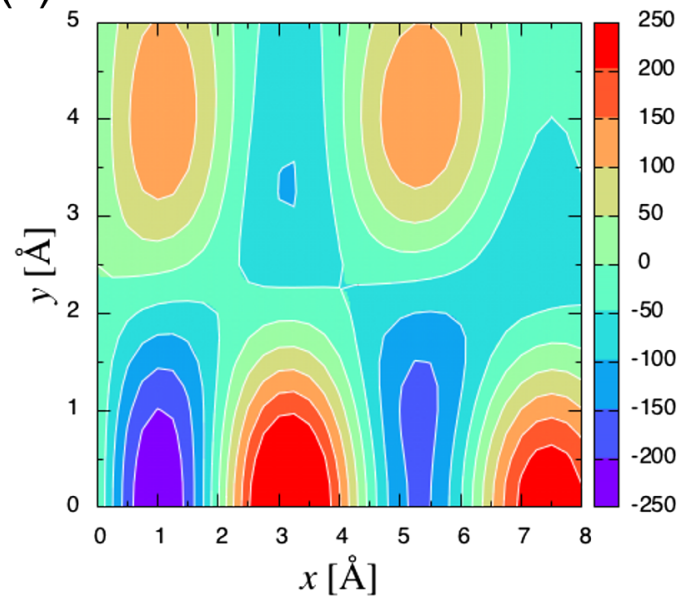

(b)

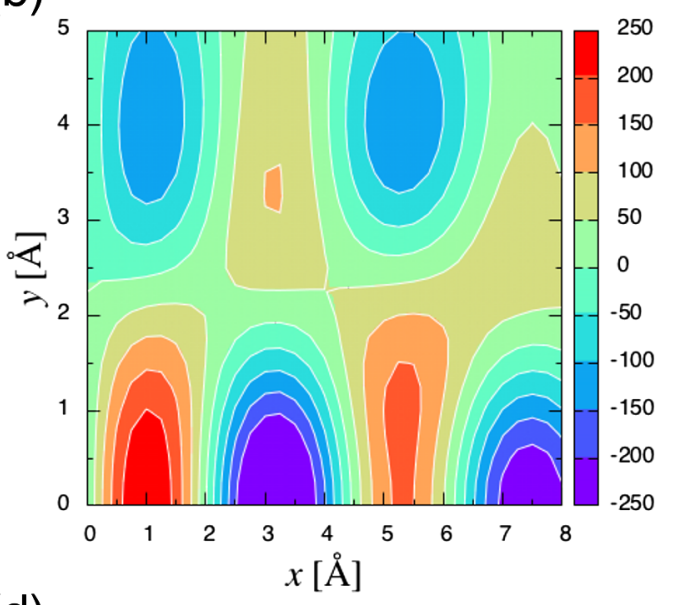

(d)

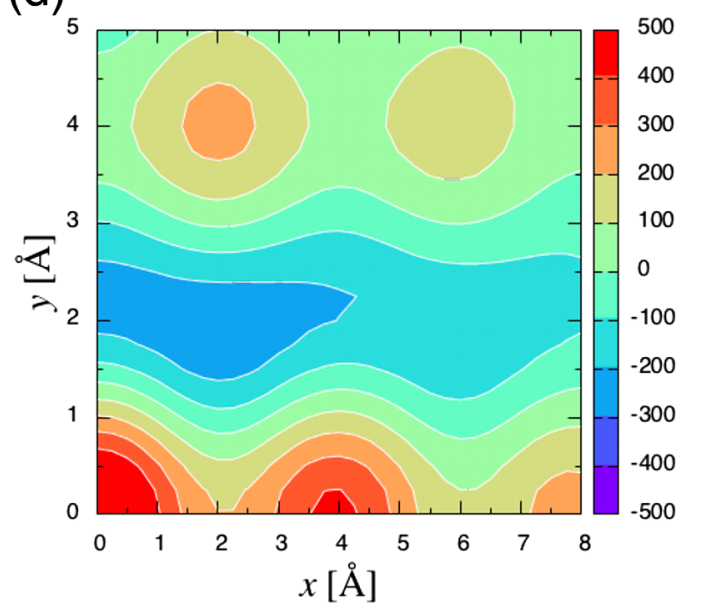

Figure 3. Calculated Fock matrix elements, $\left\langle h_{\mathrm{A}}|F| h_{\mathrm{B}}\right\rangle(\mathrm{a}),\left\langle h_{\mathrm{A}}|F| l_{\mathrm{B}}\right\rangle(\mathrm{b}),\left\langle l_{\mathrm{A}}|F| h_{\mathrm{B}}\right\rangle$ (c), and $\left\langle l_{\mathrm{A}}|F| l_{\mathrm{B}}\right\rangle$ (d), in the terrylene dimer model defined in Figure 2. The values in the color bar are given in meV.

$31 G^{*}$ basis set: the $D_{2 h}$ symmetry was imposed for both terrylene and quaterrylene. The Fock matrix of the displaced dimers was also calculated by DFT with the same functional and basis set. These quantum chemical calculations were all performed by Gaussian $09 .{ }^{37}$ The energies of $S_{1} S_{0}$ and $T_{1} T_{1}$ states of the dimer $\left(E_{\mathrm{S}_{1} \mathrm{~S}_{0}}\right.$ and $E_{\mathrm{T}_{1} \mathrm{~T}_{1}}$, respectively) are taken from our previous study on SF in oligorylenes: $E_{\mathrm{S}_{1} \mathrm{~S}_{0}}=2.29 \mathrm{eV}$ and $E_{\mathrm{T}_{1} \mathrm{~T}_{1}}=2.20 \mathrm{eV}$ for terrylene and $E_{\mathrm{S}_{1} \mathrm{~S}_{0}}=1.88 \mathrm{eV}$ and $E_{\mathrm{T}_{1} \mathrm{~T}_{1}}=$ $1.60 \mathrm{eV}$ for quaterrylene. ${ }^{20}$ These energies were all calculated on monomers under the assumption $E_{\mathrm{S}_{1} \mathrm{~S}_{0}} \approx E\left(\mathrm{~S}_{1}\right)$ and $E_{\mathrm{T}_{1} \mathrm{~T}_{1}} \approx$ $2 E\left(T_{1}\right)$ without any electronic interaction effect between monomers.

\section{RESULTS AND DISCUSSION}

3.1. Fock Matrix Elements. First, we examine the dependence of Fock matrix elements of oligorylenes on the intermolecular configurations in the dimer. Figure 3 shows the Fock matrix elements, $\left\langle h_{\mathrm{A}}|F| h_{\mathrm{B}}\right\rangle,\left\langle h_{\mathrm{A}}|F| l_{\mathrm{B}}\right\rangle,\left\langle l_{\mathrm{A}}|F| h_{\mathrm{B}}\right\rangle$, and $\left\langle l_{\mathrm{A}}|F|\right.$ $\left.l_{\mathrm{B}}\right\rangle$, in the terrylene dimer model. In the same manner as in Figure 3, Figure S1 shows the Fock matrix elements in the quaterrylene dimer model. From Figures 3 and S1 and the previous study on pentacene, ${ }^{23}$ each Fock matrix element is found to have similar node positions for these molecules because of similarity in their molecular orbital distributions. Figure 4 shows the HOMOs and LUMOs of terrylene and quaterrylene. One should pay attention to the different axis 
(a)
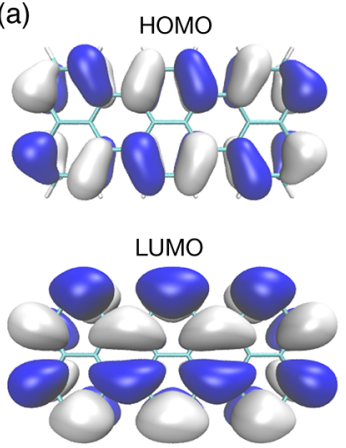

(b)
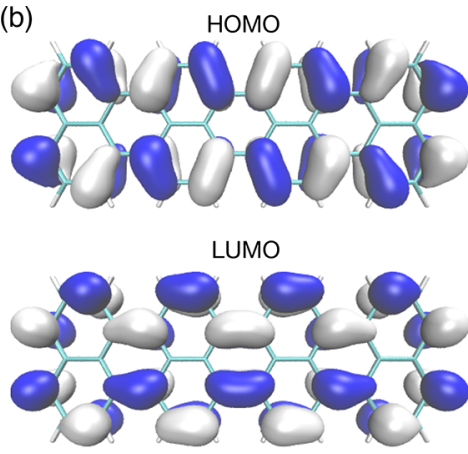

Figure 4. HOMO and LUMO of (a) terrylene and (b) quaterrylene. The white and blue surfaces indicate contours with \pm 0.02 a.u., respectively.

definitions in the present study on oligorylenes and those in ref 23 on pentacene, that is, the $x$ and $y$ axes in the present study correspond to $T$ and $L$ axes in ref 23 , respectively.

As seen from Figure $3 \mathrm{a},\left\langle h_{\mathrm{A}}|F| h_{\mathrm{B}}\right\rangle$ changes its sign along both $x$ - and $y$-slipping vectors. In slipped configurations along the $x$ axis, $\left\langle h_{\mathrm{A}}|F| h_{\mathrm{B}}\right\rangle$ takes local maxima at $x=0.0$ and $4.8 \AA$, whereas it takes local minima at $x=2.4$ and $7.2 \AA$. At the local maximum configurations, $x=0.0$ and $4.8 \AA$, it is shown that the HOMOs between the cofacial monomers maximally overlap with each other with mutually opposite phases because the terrylenes stack in the perylene unit regions (see Figure 5a,b). At the local

(a)

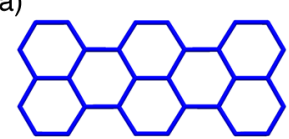

(c)

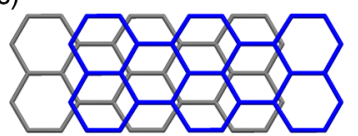

(e)

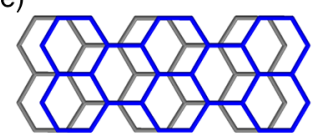

(g)

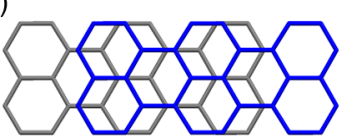

(b)

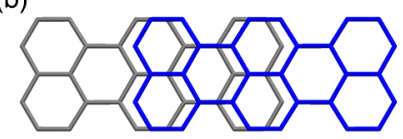

(d)

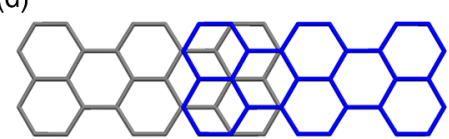

(f)

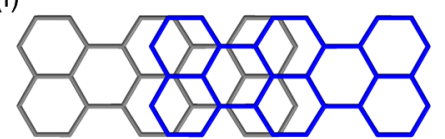

(h)

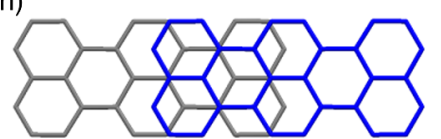

Figure 5. Terrylene dimer configurations. Local maxima of $\left\langle h_{\mathrm{A}}|F| h_{\mathrm{B}}\right\rangle$ : (a) $(x, y)=(0.0 \AA, 0.0 \AA)$ and $(\mathrm{b})(x, y)=(4.8 \AA, 0.0 \AA)$; local minima of $\left\langle h_{\mathrm{A}}|F| h_{\mathrm{B}}\right\rangle:(\mathrm{c})(x, y)=(2.4 \AA, 0.0 \AA)$ and $(\mathrm{d})(x, y)=(7.2 \AA, 0.0 \AA)$; local maxima of $\left\langle h_{\mathrm{A}}|F| l_{\mathrm{B}}\right\rangle:(\mathrm{e})(x, y)=(1.0 \AA$, $0.0 \AA)$ and $(\mathrm{f})(x, y)=(5.4$ $\AA, 0.0 \AA)$; local minima of $\left\langle h_{\mathrm{A}}|F| l_{\mathrm{B}}\right\rangle:(\mathrm{g})(x, y)=(3.2 \AA, 0.0 \AA)$; and (h) suspicious candidate packing $(x, y)=(5.8 \AA, 0.0 \AA)$. The blue and gray skeletons indicate the upper and lower terrylenes, respectively.

minimum configurations, $x=2.4$ and $7.2 \AA$, the upper and lower monomers stack by overlapping the $\pi$ orbitals at one end of each $\mathrm{C}-\mathrm{C}$ bond between the upper and lower monomers, such as stacking of the $\mathrm{C} 3$ atom of the lower monomer with the $\mathrm{C} 2$ atom of the upper one (see Figure $5 c, d$ ). In these configurations, the HOMOs between the cofacial monomers are shown to give maximal overlap with mutually opposite phases.

From Figure $3 \mathrm{~b}, \mathrm{c}$, one can apparently notice that the elements $\left\langle h_{\mathrm{A}}|F| l_{\mathrm{B}}\right\rangle$ and $\left\langle l_{\mathrm{A}}|F| h_{\mathrm{B}}\right\rangle$ have the same amplitude with mutually opposite sign at all coordinates, and this is the case with quaterrylene (see Figure $S 1$ ). This feature in $\left\langle h_{\mathrm{A}}|F| l_{\mathrm{B}}\right\rangle$ and $\left\langle l_{\mathrm{A}}|F| h_{\mathrm{B}}\right\rangle$ is also observed in the pentacene dimer model ${ }^{23}$ because of the similar periodic and symmetric HOMO and LUMO distributions of the monomer. Thus, $\left\langle l_{\mathrm{A}}|F| h_{\mathrm{B}}\right\rangle$ can be expressed as $\left\langle l_{\mathrm{A}}|F| h_{\mathrm{B}}\right\rangle=-\left\langle h_{\mathrm{A}}|F| l_{\mathrm{B}}\right\rangle$, and this relationship is found to be valid in other planar $\pi$-conjugated polycyclic hydrocarbons as long as the same displacing definition as in the present model and the symmetrical molecular structure are used. The local maximum peaks of $\left\langle h_{\mathrm{A}}|F| l_{\mathrm{B}}\right\rangle$ are found at $x=1.0$ and $5.4 \AA$, whereas that of local minimum is found at $x=3.2$ and $7.5 \AA$. In the case of $y=0.0 \AA$, the lower and upper monomers stack only with one end of each carbon(C)carbon(C) bond, such as stacking of the $\mathrm{C} 2$ atom of the lower monomer with the $\mathrm{C} 2$ atom of the upper at $(x, y)=(1.0 \AA, 0.0$ $\AA$ ) (see Figure 5e,f).

From Figure $3 \mathrm{~d}$, it can be seen that unlike $\left\langle h_{\mathrm{A}}|F| h_{\mathrm{B}}\right\rangle,\left\langle h_{\mathrm{A}}|F|\right.$ $\left.l_{\mathrm{B}}\right\rangle$, and $\left\langle l_{\mathrm{A}}|F| h_{\mathrm{B}}\right\rangle$, the element $\left\langle l_{\mathrm{A}}|F| l_{\mathrm{B}}\right\rangle$ does not show sign changes in the slips along the $x$-axis. As seen from Figure $4 \mathrm{a}, \mathrm{b}$, this can be explained by the constant phase of LUMO along the longitudinal axis of the molecule.

3.2. Effective Electronic Coupling for the SF Rate. In this section, we discuss the dependence of effective electronic coupling amplitude for the SF rate, $\mid V_{\text {eff }}$, on the intermolecular configurations. The $\left|V_{\text {eff }}\right|$ values for terrylene and quaterrylene calculated by using eq $1 \mathrm{a}$ with the Fock matrix elements and $E_{\mathrm{CT}}$ are shown in Figures 6 and S2, respectively. The variation of $E_{\mathrm{CT}}$ as a function of coordinate $(x, y)$ is shown in Figure S3.

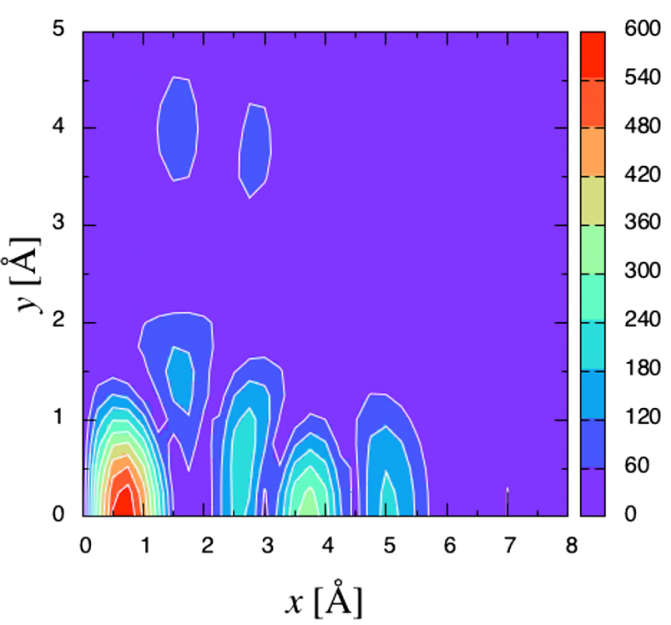

Figure 6. $I V_{\text {eff }}$ for the SF rate in terrylene. The values in the color bar are given in $\mathrm{meV}$.

As seen from Figure 6 and S2, in the $y$-axis slips of both terrylene and quaterrylene, $\left|V_{\text {eff }}\right|$ gradually decreases with increase of the $y$ value for any $x$ value. In this regard, $y$-axis slips and short-axis slips in oligorylenes are found to just contribute to weakening of $\left|V_{\text {eff }}\right|$, so that the variation of $\left|V_{\text {eff }}\right|$ by $x$-axis slips is examined. In $x$-axis slips, there are shown to be several local maximum points of $\mid V_{\text {eff }}$, for example, at $x=0.7$, 3.7, and 5.1 $\AA$. It is noted that $\left|V_{\text {eff }}\right|$ is zero at $(x, y)=(0.0 \AA, 0.0$ $\AA)$, in spite of the fact that the upper and lower monomers could maximally overlap in this configuration. As seem from Figure 3, at $(x, y)=(0.0 \AA, 0.0 \AA)$, the horizontal couplings, $\left\langle h_{\mathrm{A}}\right|$ $F\left|h_{\mathrm{B}}\right\rangle$ and $\left\langle l_{\mathrm{A}}|F| l_{\mathrm{B}}\right\rangle$, take maximum values, but the nonhorizontal 
couplings, $\left\langle h_{\mathrm{A}}|F| l_{\mathrm{B}}\right\rangle$ and $\left\langle l_{\mathrm{A}}|F| h_{\mathrm{B}}\right\rangle$, are vanished. As seen from eq 1a, therefore, $\left|V_{\text {eff }}\right|$ takes zero in the maximally overlapped configuration, $(x, y)=(0.0 \AA ̊, 0.0 \AA)$. One should pay attention that the nonadiabatic coupling between the adiabatic $S_{1} S_{0}$ and $\mathrm{T}_{1} \mathrm{~T}_{1}$ states, which is related to the transition rate from the adiabatic $S_{1} S_{0}$ to $T_{1} T_{1}$ state, at this configuration for the ethylene dimer model is not zero but $50 \%$ smaller than those in slipped-stack configurations. ${ }^{38}$

The global maximum of $\left|V_{\text {eff }}\right|$ is found to be taken in around $(x, y)=(0.7 \AA, 0.0 \AA)$, both in terrylene and quaterrylene. In this configuration, it is found that the upper and lower monomers overlap in almost the entire region, and thus both $\left\langle h_{\mathrm{A}}|F| h_{\mathrm{B}}\right\rangle$ and $\left\langle l_{\mathrm{A}}|F| l_{\mathrm{B}}\right\rangle$ take large values, though the nonhorizontal couplings, $\left\langle h_{\mathrm{A}}|F| l_{\mathrm{B}}\right\rangle$ and $\left\langle l_{\mathrm{A}}|F| h_{\mathrm{B}}\right\rangle$, are not zero. It is noted that this configuration approximately corresponds to the slip-stacked configuration with the half of a C-C bond length $(0.74 \AA$ estimated from Figure 1) along the longitudinal axis of the terrylene backbone. In the previous study in ref 2, it was pointed out that the electronic coupling related to the SF rate in the dimer models is enhanced with the slip-stacked configuration along the HOMO to LUMO transition moment. In the present model of oligorylenes, the HOMO to LUMO transition moment is placed on the longitudinal axis of terrylene or quaterrylene (see Figure 4). Therefore, the present result is in agreement with the prediction in the previous study, ${ }^{2}$ although the present molecular sizes are larger than the previous ones. In another local maximum configuration, for example, at $(x, y)=(3.7 \AA ̊ 0.0 \AA)$, the slip length along the $x$ axis approximately corresponds to the sum of the largest edge length of a benzene unit $(2.89 \AA)$ and the half of the $\mathrm{C}-\mathrm{C}$ bond length $(0.74 \AA)$. In another local maximum configuration at $(x, y)=(5.1 \AA, 0.0 \AA)$, it corresponds to the sum of the largest edge length of a benzene unit $(2.89 \AA)$ and a $\mathrm{C}-\mathrm{C}$ bond length $(1.47 \AA)$ and its half $(0.74 \AA)$. Neglecting the bond-length alternations and considering a benzene unit as a hexagonal structure, the largest edge length of a benzene unit can be regarded as twice the length of a $\mathrm{C}-\mathrm{C}$ bond. Therefore, the configurations where $\left|V_{\text {eff }}\right|$ value takes global or local maxima are considered to have a geometrical regularity, where the $\left|V_{\text {eff }}\right|-$ maximized dimer configuration is characterized by the longitudinal displacement with "the half of a $\mathrm{C}-\mathrm{C}$ bond length together with the integer multiple of the $\mathrm{C}-\mathrm{C}$ bond length in benzene units". This designing strategy of intermolecular packing for fast SF is expected to be applicable to other SF candidates of planar $\pi$-conjugated polycyclic hydrocarbons, by virtue of their common structural units of benzene. Note here that these configurations are discussed in terms of the maximum point of $\left|V_{\text {eff }}\right|$ : even in the case of the not $\left|V_{\text {eff }}\right|$ maximized configuration but of configuration giving relatively large $\left|V_{\text {eff }}\right|$, there is a possibility to undergo fast SF.

On the other hand, for avoiding smaller $\left|V_{\text {eff }}\right|$, it is not recommended to prepare oligorylene or related systems with the crystal packing of the longitudinal displacement just with "the integer multiple of the $\mathrm{C}-\mathrm{C}$ bond length in benzene units", of course including perfect stacking of $(x, y)=(0.0 \AA ̊ 0.0$ $\AA)$. As seen from Figure 4 , the phase of HOMO on each carbon atom of a longitudinal $\mathrm{C}-\mathrm{C}$ bond has an opposite sign to the other, whereas that of LUMO has the same sign between both the carbon atoms. Therefore, in these configurations, the overlap of HOMO and LUMO between monomers are predicted to be canceled out on each stacked longitudinal $\mathrm{C}-\mathrm{C}$ bond. Accordingly, in these configurations, it is predicted that $\left\langle h_{\mathrm{A}}|F| l_{\mathrm{B}}\right\rangle$ and $\left\langle l_{\mathrm{A}}|F| h_{\mathrm{B}}\right\rangle$ are vanished, resulting in the minima of $\left|V_{\text {eff }}\right|$ Indeed, peropyrene, which is a polycyclic hydrocarbon chromophore similar to oligorylenes in their monomer structures and MO distributions, was reported not to exhibit rapid $\mathrm{SF}$, where the monomers in a dimer subset of peropyrene are slip-stacked with a $\mathrm{C}-\mathrm{C}$ bond length along the longitudinal axis. $^{39}$

3.3. Perturbed Energy Levels of $S_{1} S_{0}$ and $T_{1} T_{1}$. In this section, we further analyze the results shown in the previous section by using the quasi-degenerate perturbation theory. According to this theory, we discuss the variation of energy levels related to SF because of the electronic couplings between the monomers, which provides the effective energy levels in SF. The systematic and more detailed introduction of this theory to the exciton states in SF is given in our previous study. ${ }^{40} \mathrm{We}$ here focus on the relative relationship of the perturbed energy levels of $S_{1} S_{0}$ and $T_{1} T_{1}$, the diagonal-elements in eq $2 \mathrm{a}$. As shown in eqs $2 b-2 d$, the effect of the CT states-mediated coupling is included in the perturbation energies $\left(\Delta E_{\mathrm{T}_{1} \mathrm{~T}_{1}}\right.$ and $\left.\Delta E_{S_{1} S_{0}}\right)$ and in the effective exciton-exciton coupling $J_{\text {eff }}$ between $S_{1} S_{0}$ and $S_{0} S_{1}$, which includes its perturbation term $J_{\text {CT }}$ (see eq $2 d$ ). When the mixing of CT states to $S_{1} S_{0}$ or $T_{1} T_{1}$ state is large, the perturbed $E_{\mathrm{S}_{1} \mathrm{~S}_{0}}$ or $E_{\mathrm{T}_{1} \mathrm{~T}_{1}}$ gets apart from the nonperturbed one. If the perturbed energy level of $T_{1} T_{1}$ gets below the perturbed one of $S_{1} S_{0}, S F$ is predicted to proceed exothermally, and thus SF triplet yield is expected to increase.

On the basis of the longitudinal-axis-slip-stack terrylene and quaterrylene dimer models defined in section 2.2, we discuss feasible intermolecular packing, which can realize a fast SF with a high triplet yield, that is, exothermal SF. Figure 7 shows $\left|V_{\text {eff }}\right|$ for terrylene discussed in the last section and the perturbed

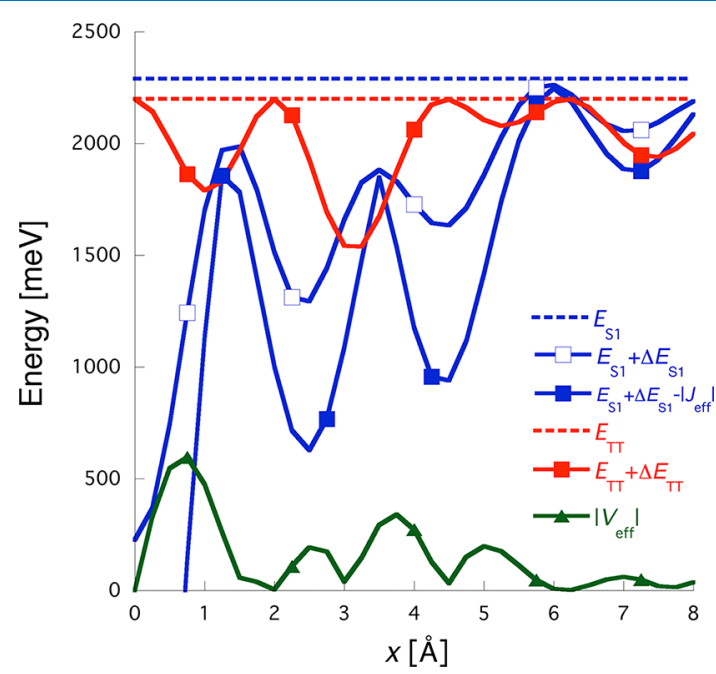

Figure 7. Perturbed energy levels of $S_{1} S_{0}$ (blue solid line) and $T_{1} T_{1}$ (red solid line) in the slipped-stack terrylene dimer model defined in Figure 2. The value of $x[\AA]$ indicates the slip length along the longitudinal axis of the molecular backbone. The dashed horizontal lines denote the nonperturbed energy levels of $E_{\mathrm{S}_{1} \mathrm{~S}_{0}}$ (blue dashed line) and $E_{\mathrm{T}_{1} \mathrm{~T}_{1}}$ (red dashed line), with the values of $E_{\mathrm{S}_{1} \mathrm{~S}_{0}}=2.29 \mathrm{eV}$ and $E_{\mathrm{T}_{1} \mathrm{~T}_{1}}$ $=2.20 \mathrm{eV}$, respectively. ${ }^{20}$ The two blue solid lines denote the perturbed $E_{\mathrm{S}_{1} \mathrm{~S}_{0}}, E_{\mathrm{S}_{1} \mathrm{~S}_{0}}+\Delta E_{\mathrm{S}_{1} \mathrm{~S}_{0}}$ (with white squares), and the possible lowest split energy of $E_{\mathrm{S}_{1} \mathrm{~S}_{\mathrm{o}^{\prime}}} E_{\mathrm{S}_{1} \mathrm{~S}_{0}}+\Delta E_{\mathrm{S}_{1} \mathrm{~S}_{0}}-J_{\text {eff }} \mathrm{I}$ (with blue squares). The red solid line denotes the perturbed $E_{\mathrm{T}_{1} \mathrm{~T}_{1}}$ and $E_{\mathrm{T}_{1} \mathrm{~T}_{1}}+\Delta E_{\mathrm{T}_{1} \mathrm{~T}_{1}}$. The green solid line denotes the $\left|V_{\text {eff }}\right|$ value in the corresponding slippedstack configuration. 
energy levels, $E_{\mathrm{S}_{1} \mathrm{~S}_{0}}+\Delta E_{\mathrm{S}_{1} \mathrm{~S}_{0}}$ and $E_{\mathrm{T}_{1} \mathrm{~T}_{1}}+\Delta E_{\mathrm{T}_{1} \mathrm{~T}_{1}}$, and $E_{\mathrm{S}_{1} \mathrm{~S}_{0}}+$ $\Delta E_{\mathrm{S}_{1} \mathrm{~S}_{0}}$ split by the effective exciton-exciton coupling $J_{\text {eff }}$, where we consider the possible lowest split energy level, $E_{\mathrm{S}_{1} \mathrm{~S}_{0}}+\Delta E_{\mathrm{S}_{1} \mathrm{~S}_{0}}$ - $J_{\text {eff }}$. These quantities for quaterrylene are shown in Figure S4. Because $E_{\mathrm{S}_{1} \mathrm{~S}_{0}}$ and $E_{\mathrm{T}_{1} \mathrm{~T}_{1}}$ are smaller than $E_{\mathrm{CT}}$ in the present systems and the numerators in eqs $2 b$ and $2 c$ are given in positive values, the energy perturbation terms $\Delta E_{\mathrm{S}_{1} S_{0}}$ and $\Delta E_{\mathrm{T}_{1} \mathrm{~T}_{1}}$ are shown to be negative and are expected to stabilize the nonperturbed $E_{\mathrm{S}_{1} \mathrm{~S}_{0}}$ and $E_{\mathrm{T}_{1} \mathrm{~T}_{1}}$, respectively. As seen from Figure 7 , for terrylene in all $x$-axis-slipped dimer configurations, the energy levels of the nonperturbed $S_{1} S_{0}$ and $T_{1} T_{1}$ are found to be swayed and lowered by the perturbation terms $\Delta E_{\mathrm{S}_{1} \mathrm{~S}_{0}}$ and $\Delta E_{\mathrm{T}_{1} \mathrm{~T}_{1}}$, respectively, because of the individual variation of the Fock matrix elements. The effect of $\left|J_{\text {eff }}\right|$ on the energy splitting between $S_{1} S_{0}$ and $S_{0} S_{1}$ is found to be crucial for expecting exothermal SF, that is, $J_{\text {eff }}$ makes much lower the level of $E_{S_{1} S_{0}}$ $+\Delta E_{\mathrm{S}_{1} \mathrm{~S}_{0}}$ especially in the configurations where $E_{\mathrm{S}_{1} \mathrm{~S}_{0}}+\Delta E_{\mathrm{S}_{1} \mathrm{~S}_{0}}$ takes minima. Therefore, from the viewpoint of generating more triplet excitons, it is indispensable to weaken the impact of $\left|J_{\text {eff }}\right|$ as well as $\Delta E_{\mathrm{S}_{1}}$ by optimizing intermolecular packing. Unfortunately, as is the case with pentacene dimers, ${ }^{40}$ the stabilized energy levels of $S_{1} S_{0}$ seems to be overestimated due to large horizontal Fock matrix elements in small $x$ regions $(x<$ $1.0 \AA$ ), where $E_{\mathrm{S}_{1} \mathrm{~S}_{0}}+\Delta E_{\mathrm{S}_{1} \mathrm{~S}_{0}}$ fall below $E_{\mathrm{T}_{1} \mathrm{~T}_{1}}+\Delta E_{\mathrm{T}_{1} \mathrm{~T}_{1}}$ by more than $1000 \mathrm{meV}$. At any rate, in these largely overlapped configurations of chromophores, the excimer formation is predicted to occur because of the largely stabilized $S_{1}$ energy level, the feature of which prevents the creation of the doubletriplet exciton. Such an excimer formation caused by the significantly stabilized $S_{1}$ energy level is also observed in the real crystal of cofacially stacked simplest oligorylene, perylene. ${ }^{41}$ On the other hand, it turns out that at $x=1.2 \AA$ and $3.3 \AA$, both $E_{\mathrm{S}_{1} \mathrm{~S}_{0}}+\Delta E_{\mathrm{S}_{1} \mathrm{~S}_{0}}$ and $E_{\mathrm{S}_{1} \mathrm{~S}_{0}}+\Delta E_{\mathrm{S}_{1} \mathrm{~S}_{0}}-J_{\text {eff }}$ take maxima, whereas $E_{\mathrm{T}_{1} \mathrm{~T}_{1}}+\Delta E_{\mathrm{T}_{1} \mathrm{~T}_{1}}$ takes minima. It is consequently found that around $x=1.2$ and $3.3 \AA$, $E_{\mathrm{T}_{1} \mathrm{~T}_{1}}+\Delta E_{\mathrm{T}_{1} \mathrm{~T}_{1}}$ gets lower than $E_{\mathrm{S}_{1} \mathrm{~S}_{0}}+$ $\Delta E_{\mathrm{S}_{1} \mathrm{~S}_{0}}$, and thus exothermal SF can be expected. It is also noted that $\left|V_{\text {eff }}\right|$ takes relatively large values in these dimer configurations, about $270 \mathrm{meV}$ for $x=1.2 \AA$ and $250 \mathrm{meV}$ for $x=3.3 \AA$. Combining the results of the estimated $\left|V_{\text {eff }}\right|$ and the perturbed exciton-state energies, it can be expected that a fast and exothermal SF coexists in these dimer configurations, that is, at $x=1.2$ and $3.3 \AA$ (see Figure 5e,g). Considering overlaps of the existing terrylene derivatives 42 and slip-stacked crystal packing in $\pi$-conjugated polycyclic hydrocarbons, ${ }^{43,44}$ these dimer configurations are expected to be realized. These energy levels are estimated based on the perturbation theory, which allows us to illustrate effective SF energetics in a simple way based on electronic coupling. In addition, when the dimer subset is embedded in the crystalline bulk, the CT energy is predicted to be stabilized due to polarizations of the surrounding molecules. ${ }^{45}$ Thus, the present effective energy levels would somewhat differ from those in the realistic bulk crystal. We have estimated the effect of this stabilization by examining the effective energy levels for models with evenly decreased $E_{\text {Ст }}$. As a result, it is found that the relative relationship between the perturbed $S_{1} S_{0}$ and $T_{1} T_{1}$ energy levels is not changed, though the absolute value of each energy level is significantly changed (see Figure S5). This implies that the present conclusions are qualitatively valid even in the realistic bulk crystal.

For quaterrylene in Figure S4, the variation features of the perturbed exciton-state energies for the molecular displacement are shown to be similar to those of terrylene, so that the candidate packings for efficient SF in quaterrylene is found to be the same as that in terrylene with $x=1.2$ and $3.3 \AA$. In addition, as seen from Figures 7 and S4, around $x=5.8 \AA$ for terrylene and $x=5.5 \AA$ for quatterylene, the perturbed energy level of $S_{1} S_{0}$ is apparently higher than that of $T_{1} T_{1}$. However, in terrylene with $x=5.8 \AA$, the $\left|V_{\text {eff }}\right|$ value is shown to be small (47 $\mathrm{meV}$ ), and the overlap area between the monomers is predicted to be too small to configure realistic crystal packing in such $\pi$ conjugated systems (see Figure $5 \mathrm{~h}$ ), so that an efficient SF is less expected in this dimer configuration. On the other hand, it is found that in quaterrylene with $x=5.5 \AA$, $\left|V_{\text {eff }}\right|$ takes a relatively large value $(123 \mathrm{meV})$, and the overlap area is relatively large (see Figure S6), so that this configuration is feasible and is expected to be a candidate packing for efficient $\mathrm{SF}$ in quaterrylene in addition to the configurations $x=1.2$ and $3.3 \AA ̊$.

3.4. Application to Real Crystal Packings of Terrylenes. In this section, we apply the present method for the evaluation of two types of dimer subsets in the crystal structures of terrylene derivatives, 2,5,10,13-tetra(tert-butyl)terrylene (1) and 2,5-di(tert-butyl)terrylene (2). As mentioned in the introduction, it was reported that $\mathbf{1}$ and $\mathbf{2}$ have different stacking geometries, SF rates, and triplet yields, where the observed SF in $\mathbf{1}$ was faster and gave a smaller triplet yield than 2. $^{21}$ The structural data available for constructing these configurations were taken from ref 21. Their packing configurations are shown in Figure S7.

Table 1 lists the calculated result of Fock matrix elements, $J_{\text {Coul }}$ and $E_{\mathrm{CT}}$, for the dimer subsets in $\mathbf{1}$ and 2 . The comparison

Table 1. Calculated Fock Matrix Elements, $J_{\text {Coul }}$ and $E_{\mathrm{CT}}$ Values $[\mathrm{meV}]$ for the Dimer Subsets of 1 and 2

$\begin{array}{lll}\text { system } & \mathbf{1} & \mathbf{2} \\ \left\langle h_{\mathrm{A}}|F| h_{\mathrm{B}}\right\rangle & -152.03 & 190.42 \\ \left\langle h_{\mathrm{A}}|F| l_{\mathrm{B}}\right\rangle & 67.78 & 90.31 \\ \left\langle l_{\mathrm{A}}|F| h_{\mathrm{B}}\right\rangle & -57.27 & 91.74 \\ \left\langle l_{\mathrm{A}}|F| l_{\mathrm{B}}\right\rangle & 46.04 & 86.15 \\ J_{\text {Coul }} & -41.74 & -7.29 \\ E_{\text {CT }} & 2544 & 2755\end{array}$

of $\left|V_{\text {eff }}\right|$ calculated from these elements is shown in Table 2. As seen from Table 1, the absolute value of each Fock matrix element of $\mathbf{2}$ is found to be larger than that of $\mathbf{1}$ because of the smaller interplanar distance between monomers of $\mathbf{2}$ than that of $\mathbf{1}$. Thus, the electronic coupling of $\mathbf{2}$ seems to be larger than that of 1 , but in fact, $\left|V_{\text {eff }}\right|$ for $\mathbf{1}$ is found to be larger than that for 2 , as seen from Table 2 . This can be explained as follows. As seen from Table 1 , the nonhorizontal couplings, $\left\langle h_{\mathrm{A}}|F| l_{\mathrm{B}}\right\rangle$ and $\left\langle l_{\mathrm{A}}|F| h_{\mathrm{B}}\right\rangle$, have the opposite sign in $\mathbf{1}$, whereas in 2 , they have the same sign. This opposite sign relation of these nonhorizontal couplings between $\mathbf{1}$ and $\mathbf{2}$ is understood by considering the different molecular orbital (MO) overlapping for the nonhorizontal couplings in 2, which includes a monomer stacked with $180^{\circ}$ rotation around the short axis of the molecule in $\mathbf{2}$ (see Supporting Information). It is found that according to the different $\mathrm{MO}$ phase relationship in 2, the amplitudes of $\left|V_{\text {eff }}\right|$ for $\mathbf{1}$ and $\mathbf{2}$ are not only straightforwardly 
Table 2. Effective Electronic Coupling $\left(\left|V_{\text {eff }}\right|\right)$, Perturbed Energy Levels of $S_{1} S_{0}$ and $T_{1} T_{1}$, and Experimental SF Timescales and Triplet Yields in the Crystal Packings 1 and 2 in Ref 21

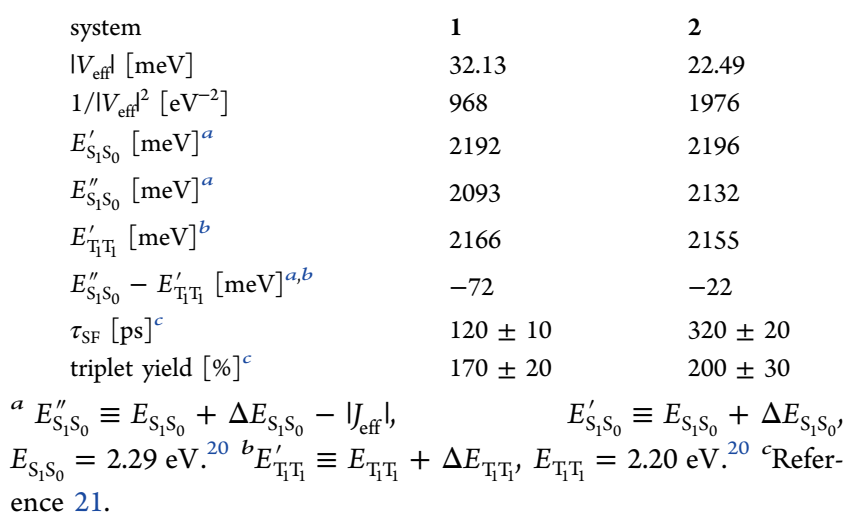

estimated from the difference of interplanar distances but are also obtained from the combination of each element in eq 1 a. As a result, the $\left|V_{\text {eff }}\right|$ value for $\mathbf{1}$ is shown to be larger than that for 2. The SF rate is characterized by the SF timescale $\tau_{\mathrm{SF}}$, which is proportional to the inverse of the square of the $\left|V_{\text {eff }}\right|$ value, $\tau_{\mathrm{SF}} \propto 1 / \mid V_{\text {eff }}{ }^{2}$. By comparing the $1 /\left|V_{\text {eff }}\right|^{2}$ values and the experimental $\tau_{\mathrm{SF}}$ values summarized in Table 2 , the calculated I $V_{\text {effl }}$ values for $\mathbf{1}$ and $\mathbf{2}$ are shown to reproduce the experimental results qualitatively. Judging from this good agreement with the experiment, the present evaluation scheme of $\left|V_{\text {eff }}\right|$ is concluded to be a useful method for investigating the effect of intermolecular packing on the SF rate and searching for the packing forms yielding large SF rates, at least under the comparisons in the same chromophore dimer subset.

On the other hand, as for the effective energetics of $\mathbf{1}$ and $\mathbf{2}$ in $\mathrm{SF}$, the perturbed energy levels of $\mathrm{S}_{1} \mathrm{~S}_{0}$ (denoted as $E_{\mathrm{S}_{1} \mathrm{~S}_{0}}^{\prime}$ excluding the energy splitting by $J_{\text {eff }} \mid$ and as $E_{S_{1} S_{0}}^{\prime \prime}$ including it), $\mathrm{T}_{1} \mathrm{~T}_{1}$ (denoted as $E_{\mathrm{T}_{1} \mathrm{~T}_{1}}^{\prime}$ ), and their effective difference (denoted as $\left.E_{\mathrm{S}_{1} \mathrm{~S}_{0}}^{\prime \prime}-E_{\mathrm{T}_{1} \mathrm{~T}_{1}}^{\prime}\right)$ are shown in Table 2. The $E_{\mathrm{S}_{1} \mathrm{~S}_{0}}^{\prime \prime}-E_{\mathrm{T}_{1} \mathrm{~T}_{1}}^{\prime}$ values both in 1 and 2 are shown to be negative with -72 and -22 $\mathrm{meV}$, respectively; that is, it is expected that SF occurs endothermically both in $\mathbf{1}$ and $\mathbf{2}$ and more endothermically in $\mathbf{1}$ than in 2. This difference of the effective energy levels between $\mathbf{1}$ and $\mathbf{2}$ is expected to explain the different experimental triplet yields between them. As seen from eqs $2 b$ and $2 c$ and the values shown in Table 1 , the perturbation energies $\left(\Delta E_{\mathrm{T}_{1} \mathrm{~T}_{1}}\right.$ and $\left.\Delta E_{\mathrm{S}_{1} \mathrm{~S}_{0}}\right)$ do not affect crucially the difference of energy levels of $S_{1} S_{0}$ and $T_{1} T_{1}$ between 1 and 2 ; in fact, the differences of $E_{S_{1} S_{0}}^{\prime}$ and $E_{\mathrm{T}_{1} \mathrm{~T}_{1}}^{\prime}$ between 1 and 2 are calculated to be only 4 and 11 $\mathrm{meV}$, respectively. Thus, the origin of their different effective energy levels is predicted to lie in the effective excitonic coupling $\mid J_{\text {eff }}$. From eq $2 \mathrm{~d}$ and the values shown in Table 1 , the signs of $J_{\text {Coul }}$ are shown to be negative both in $\mathbf{1}$ and $\mathbf{2}$, and the sign of $J_{\mathrm{CT}}$ is shown to be negative in $\mathbf{1}$ while positive in 2 . It is therefore found that in the case of $\mathbf{1}$, the amplitude of $J_{\text {eff }}$ gets constructively larger, whereas in the case of 2 , the amplitude of $\mid J_{\text {eff }}$ gets smaller because of the opposite sign of $J_{\text {Coul }}$ and $J_{\mathrm{CT}}$. It is noted that although $E_{\mathrm{S}_{1} \mathrm{~S}_{0}}^{\prime \prime}-E_{\mathrm{T}_{1} \mathrm{~T}_{1}}^{\prime}$ takes negative values both in $\mathbf{1}$ and $\mathbf{2}$ and hence their SF are expected to proceed endothermically, the triplet yields observed in the experiment are large enough $\left(170 \pm 20\right.$ and $200 \pm 30 \%$, respectively $\left.{ }^{21}\right)$, and these large triplet yields in their real crystal are more or less predicted to originate in the close $E_{\mathrm{S}_{1} \mathrm{~S}_{0}}^{\prime \prime}$ and $E_{\mathrm{T}_{1} \mathrm{~T}_{1}}^{\prime}$ levels.

In the literature for $\mathbf{1}$ and $\mathbf{2}$, the energetics estimated from the absorption spectroscopies was concluded to be the same, $E_{\mathrm{S}_{1} \mathrm{~S}_{0}}=1.93 \mathrm{eV}$ and $E_{\mathrm{T}_{1} \mathrm{~T}_{1}}=2.00 \mathrm{eV},{ }^{21}$ so that the different $\mathrm{SF}$ performances that originated from different crystal packings of 1 and $\mathbf{2}$ were not fully explained only by such observables. As shown in this study, the consideration of effective energetics in the SF process, which is not obtained by the above absorption spectroscopy, including the effect of CT-mixing of intermolecular electronic couplings is indispensable for understanding the SF rate and triplet yield. As a result, the proposed method is shown to complement the experimental results and explain successfully both for the SF rate and SF triplet yield. We here note that the present methodology does not calculate their real values. Therefore, other factors such as the external effect on the dimer and the vibronic coupling should be taken into consideration for a more detailed discussion. Nevertheless, electronic couplings and the relative effective energy levels have been elucidated to be important factors for the difference in the SF rate and triplet yield.

Finally, we compare the features of SF between the candidate packings proposed in section 3.3 and the experimental ones for 1 and 2. We can expect further faster SF and larger SF triplet yields in those candidate packings than in $\mathbf{1}$ and $\mathbf{2}$ packings. This is understood as follows. In the candidate packings, $\left|V_{\text {eff }}\right|$ values are shown to be 1 order of magnitude larger than those calculated in 1 and 2 packings, which leads to 2 or more orders of magnitude faster SF than 1 and $\mathbf{2}$ packings, and the effective energetics for the present candidates implies exothermic SF, though 1 and 2 packings were reported to be endothermic. In addition, $\left|V_{\text {eff }}\right|$ values in the present candidate packings for oligorylenes are found to be larger than previously reported electronic couplings in the real crystals of polyacenes, though I $V_{\text {eff }}$ is not exactly the same quantity as their electronic couplings. ${ }^{44}$ Therefore, the SF rates of the present candidate packings of oligorylenes have a possibility to exceed those of well-studied systems such as tetracene and pentacene.

\section{CONCLUSIONS}

We have investigated the SF rate and the effective energetics in SF based on the analysis of the electronic couplings of two types of oligorylenes, terrylene and quaterrylene. For the SF rate, we have estimated the effective electronic couplings $\left(\left|V_{\text {eff }}\right|\right)$ using the related electronic couplings for various packing forms. $\left|V_{\text {eff }}\right|$ in the present model has local maxima in several longitudinal slip-stacked configurations, where fast SF can be expected. On the other hand, for the effective energetics in SF, we have analyzed the variation of energy levels of the perturbed $\mathrm{S}_{1} \mathrm{~S}_{0}$ and $\mathrm{T}_{1} \mathrm{~T}_{1}$ states, particularly in the longitudinal slipstacked oligorylenes. The effective energy gap between the perturbed $S_{1} S_{0}$ and $T_{1} T_{1}$ states has been revealed to drastically change by the modification of relative configurations between the monomers. Combining the results of $\left|V_{\text {eff }}\right|$ and the effective energetics in SF, we have proposed several candidate molecular packing forms for a more efficient SF in oligorylene systems. Moreover, the present method has been shown to explain a qualitative difference in SF performances of the two real crystal packings of terrylene. Therefore, the present method contributes to realizing the rational design of efficient SF materials by controlling the intermolecular packing, that is, crystal engineering. The present results will stimulate both 
theoretical investigations and experimental crystal engineering for preparing highly efficient SF materials, though there still remains unexplored issues on SF in oligorylenes, such as vibronic coupling effects ${ }^{46,47}$ and exciton dynamics. ${ }^{22-24,48-50}$ In addition to these aspects, the assessment of applicability of the present simpler adiabatic model to a realistic multiconfigurational model ${ }^{13,51,52}$ and the theoretical treatment of morphology in the condensed phase for $\mathrm{SF}^{53-55}$ are indispensable for the future comprehensive understanding of SF.

\section{ASSOCIATED CONTENT}

\section{S Supporting Information}

The Supporting Information is available free of charge on the ACS Publications website at DOI: 10.1021/acsomega.7b00655.

Details of the effective electronic coupling for the SF rate and the effective Hamiltonian; Fock matrix elements in quaterrylene; effective electronic coupling for the SF rate in quaterrylene; energies of CT states in terrylene and quaterrylene; diagram of perturbed energy levels for quaterrylene; diagram of perturbed energy levels of terrylene with the assumption that $E_{\mathrm{CT}}$ is stabilized by $250 \mathrm{meV}$; candidate packing for efficient $\mathrm{SF}$ in quaterrylene; crystal packing patterns of terrylene derivatives; and nonhorizontal couplings in the present model and in 2,5-di(tert-butyl)terrylene (PDF)

\section{AUTHOR INFORMATION}

\section{Corresponding Author}

*E-mail: mnaka@cheng.es.osaka-u.ac.jp.

ORCID

Takanori Nagami: 0000-0001-9252-0596

Soichi Ito: 0000-0001-6550-702X

Masayoshi Nakano: 0000-0002-3544-1290

Notes

The authors declare no competing financial interest.

\section{ACKNOWLEDGMENTS}

This work was supported by JSPS KAKENHI grant number JP2645050 in JSPS Research Fellowship for Young Scientists, grant number JP25248007 in Scientific Research (A), grant number JP24109002 in Scientific Research on Innovative Areas "Stimuli-Responsive Chemical Species", grant numbers JP15H00999 and JP17H05157 in Scientific Research on Innovative Areas " $\pi$-System Figuration", and grant numbers JP26107004 and JP15H01086 in Scientific Research on Innovative Areas "Photosynergetics". This is also partly supported by King Khalid University through a grant RCAMS/KKU/001-16 under the Research Center for Advanced Materials Science at the King Khalid University, Kingdom of Saudi Arabia. Theoretical calculations were partly performed in the Research Center for Computational Science, Okazaki, Japan.

\section{REFERENCES}

(1) Smith, M. B.; Michl, J. Singlet Fission. Chem. Rev. 2010, 110, 6891-6936.

(2) Smith, M. B.; Michl, J. Recent Advances in Singlet Fission. Annu. Rev. Phys. Chem. 2013, 64, 361-386.

(3) Hanna, M. C.; Nozik, A. J. Solar Conversion Efficiency of Photovoltaic and Photoelectrolysis Cells with Carrier Multiplication Absorbers. J. Appl. Phys. 2006, 100, 074510.
(4) Liu, Y.; Zhang, C.; Wang, R.; Zhang, B.; Tan, Z.; Wang, X.; Xiao, M. Large Optical Nonlinearity Induced by Singlet Fission in Pentacene Films. Angew. Chem., Int. Ed. 2015, 54, 6222-6226.

(5) Zhao, M.; Liu, K.; Zhang, Y.-D.; Wang, Q.; Li, Z.-G.; Song, Y.-L.; Zhang, H.-L. Singlet Fission Induced Giant Optical Limiting Responses of Pentacene Derivatives. Mater. Horiz. 2015, 2, 619-624.

(6) Herz, J.; Buckup, T.; Paulus, F.; Engelhart, J.; Bunz, U. H. F.; Motzkus, M. Acceleration of Singlet Fission in an Aza-Derivative of TIPS-Pentacene. J. Phys. Chem. Lett. 2014, 5, 2425-2430.

(7) Johnson, J. C.; Nozik, A. J.; Michl, J. The Role of Chromophore Coupling in Singlet Fission. Acc. Chem. Res. 2013, 46, 1290-1299.

(8) Eaton, S. W.; Shoer, L. E.; Karlen, S. D.; Dyar, S. M.; Margulies, E. A.; Veldkamp, B. S.; Ramanan, C.; Hartzler, D. A.; Savikhin, S.; Marks, T. J.; et al. Singlet Exciton Fission in Polycrystalline Thin Films of a Slip-Stacked Perylenediimide. J. Am. Chem. Soc. 2013, 135, 14701-14712.

(9) Kato, D.; Sakai, H.; Tkachenko, N. V.; Hasobe, T. High-Yield Excited Triplet States in Pentacene Self-Assembled Monolayers on Gold Nanoparticles through Singlet Exciton Fission. Angew. Chem., Int. Ed. 2016, 55, 5230-5234.

(10) Busby, E.; Berkelbach, T. C.; Kumar, B.; Chernikov, A.; Zhong, Y.; Hlaing, H.; Zhu, X.-Y.; Heinz, T. F.; Hybertsen, M. S.; Sfeir, M. Y.; et al. Multiphonon Relaxation Slows Singlet Fission in Crystalline Hexacene. J. Am. Chem. Soc. 2014, 136, 10654-10660.

(11) Berkelbach, T. C.; Hybertsen, M. S.; Reichman, D. R Microscopic Theory of Singlet Exciton Fission. I. General Formulation. J. Chem. Phys. 2013, 138, 114102.

(12) Zimmerman, P. M.; Bell, F.; Casanova, D.; Head-Gordon, M. Mechanism for Singlet Fission in Pentacene and Tetracene: From Single Exciton to Two Triplets. J. Am. Chem. Soc. 2011, 133, 1994419952.

(13) Feng, X.; Luzanov, A. V.; Krylov, A. I. Fission of Entangled Spins: An Electronic Structure Perspective. J. Phys. Chem. Lett. 2013, 4, 3845-3852.

(14) Feng, X.; Krylov, A. I. On Couplings and Excimers: Lessons from Studies of Singlet Fission in Covalently Linked Tetracene Dimers. Phys. Chem. Chem. Phys. 2016, 18, 7751-7761.

(15) Minami, T.; Nakano, M. Diradical Character View of Singlet Fission. J. Phys. Chem. Lett. 2012, 3, 145-150.

(16) Minami, T.; Ito, S.; Nakano, M. Fundamental of DiradicalCharacter-Based Molecular Design for Singlet Fission. J. Phys. Chem. Lett. 2013, 4, 2133-2137.

(17) Nakano, M. Open-Shell-Character-Based Molecular Design Principles: Applications to Nonlinear Optics and Singlet Fission. Chem. Rec. 2017, 17, 27-62.

(18) Paci, I.; Johnson, J. C.; Chen, X.; Rana, G.; Popović, D.; David, D. E.; Nozik, A. J.; Ratner, M. A.; Michl, J. Singlet Fission for DyeSensitized Solar Cells: Can a Suitable Sensitizer Be Found? J. Am. Chem. Soc. 2006, 128, 16546-16553.

(19) Nakano, M.; Fukui, H.; Minami, T.; Yoneda, K.; Shigeta, Y.; Kishi, R.; Champagne, B.; Botek, E.; Kubo, T.; Ohta, K.; et al. (Hyper)polarizability Density Analysis for Open-Shell Molecular Systems Based on Natural Orbitals and Occupation Numbers. Theor. Chem. Acc. 2011, 130, 711-724.

(20) Minami, T.; Ito, S.; Nakano, M. Theoretical Study of Singlet Fission in Oligorylenes. J. Phys. Chem. Lett. 2012, 3, 2719-2723.

(21) Eaton, S. W.; Miller, S. A.; Margulies, E. A.; Shoer, L. E.; Schaller, R. D.; Wasielewski, M. R. Singlet Exciton Fission in Thin Films of tert-Butyl-Substituted Terrylenes. J. Phys. Chem. A 2015, 119, $4151-4161$

(22) Renaud, N.; Grozema, F. C. Intermolecular Vibrational Modes Speed up Singlet Fission in Perylenediimide Crystals. J. Phys. Chem. Lett. 2015, 6, 360-365.

(23) Wang, L.; Olivier, Y.; Prezhdo, O. V.; Beljonne, D. Maximizing Singlet Fission by Intermolecular Packing. J. Phys. Chem. Lett. 2014, 5, 3345-3353.

(24) Mirjani, F.; Renaud, N.; Gorczak, N.; Grozema, F. C. Theoretical Investigation of Singlet Fission in Molecular Dimers: 
The Role of Charge Transfer States and Quantum Interference. J. Phys. Chem. C 2014, 118, 14192-14199.

(25) Renaud, N.; Sherratt, P. A.; Ratner, M. A. Mapping the Relation between Stacking Geometries and Singlet Fission Yield in a Class of Organic Crystals. J. Phys. Chem. Lett. 2013, 4, 1065-1069.

(26) Havlas, Z.; Michl, J. Guidance for Mutual Disposition of Chromophores for Singlet Fission. Isr. J. Chem. 2016, 56, 96-106.

(27) Zirzlmeier, J.; Lehnherr, D.; Coto, P. B.; Chernick, E. T.; Casillas, R.; Basel, B. S.; Thoss, M.; Tykwinski, R. R.; Guldi, D. M. Singlet Fission in Pentacene Dimers. Proc. Natl. Acad. Sci. U.S.A. 2015, $112,5325-5330$.

(28) Korovina, N. V.; Das, S.; Nett, Z.; Feng, X.; Joy, J.; Haiges, R.; Krylov, A. I.; Bradforth, S. E.; Thompson, M. E. Singlet Fission in a Covalently Linked Cofacial Alkynyltetracene Dimer. J. Am. Chem. Soc. 2016, 138, 617-627.

(29) Ito, S.; Nagami, T.; Nakano, M. Design Principles of Electronic Couplings for Intramolecular Singlet Fission in Covalently-Linked Systems. J. Phys. Chem. A 2016, 120, 6236-6241.

(30) Sakuma, T.; Sakai, H.; Araki, Y.; Mori, T.; Wada, T.; Tkachenko, N. V.; Hasobe, T. Long-Lived Triplet Excited States of Bent-Shaped Pentacene Dimers by Intramolecular Singlet Fission. J. Phys. Chem. A 2016, 120, 1867-1875.

(31) Wu, Y.; Liu, K.; Liu, H.; Zhang, Y.; Zhang, H.; Yao, J.; Fu, H. Impact of Intermolecular Distance on Singlet Fission in a Series of TIPS Pentacene Compounds. J. Phys. Chem. Lett. 2014, 5, 3451-3455.

(32) Zimmerman, P. M.; Zhang, Z.; Musgrave, C. B. Singlet Fission in Pentacene through Multi-Exciton Quantum States. Nat. Chem. 2010, 2, 648-652.

(33) Shavitt, I.; Redmon, L. T. Quasidegenerate Perturbation Theories. A Canonical van Vleck Formalism and Its Relationship to Other Approaches. J. Chem. Phys. 1980, 73, 5711-5717.

(34) Casanova, D. Electronic Structure Study of Singlet Fission in Tetracene Derivatives. J. Chem. Theory Comput. 2014, 10, 324-334.

(35) Vallett, P. J.; Snyder, J. L.; Damrauer, N. H. Tunable Electronic Coupling and Driving Force in Structurally Well-Defined Tetracene Dimers for Molecular Singlet Fission: A Computational Exploration Using Density Functional Theory. J. Phys. Chem. A 2013, 117, 1082410838 .

(36) Kerr, K. A.; Ashmore, J. P.; Speakman, J. C. The Crystal and Molecular Structure of Quaterrylene: A Redetermination. Proc. R. Soc. London, Ser. A 1975, 344, 199-215.

(37) Frisch, M. J.; Trucks, G. W.; Schlegel, H. B.; Scuseria, G. E.; Robb, M. A.; Cheeseman, J. R.; Scalmani, G.; Barone, V.; Mennucci, B.; Petersson, G. A.; et al. Gaussian 09, Revision B.01; Gaussian, 2010; p 2009.

(38) Matsika, S.; Feng, X.; Luzanov, A. V.; Krylov, A. I. What We Can Learn from the Norms of One-Particle Density Matrices, and What We Can't: Some Results for Interstate Properties in Model Singlet Fission Systems. J. Phys. Chem. A 2014, 118, 11943-11955.

(39) Nichols, V. M.; Rodriguez, M. T.; Piland, G. B.; Tham, F.; Nesterov, V. N.; Youngblood, W. J.; Bardeen, C. J. Assessing the Potential of Peropyrene as a Singlet Fission Material: Photophysical Properties in Solution and the Solid State. J. Phys. Chem. C 2013, 117, 16802-16810.

(40) Ito, S.; Nagami, T.; Nakano, M. Singlet Fission in PancakeBonded Systems. Phys. Chem. Chem. Phys. 2017, 19, 5737-5745.

(41) Margulies, E. A.; Shoer, L. E.; Eaton, S. W.; Wasielewski, M. R. Excimer Formation in Cofacial and Slip-Stacked Perylene-3,4:9,10Bis(dicarboximide) Dimers on a Redox-Inactive Triptycene Scaffold. Phys. Chem. Chem. Phys. 2014, 16, 23735-23742.

(42) Margulies, E. A.; Miller, C. E.; Wu, Y.; Ma, L.; Schatz, G. C.; Young, R. M.; Wasielewski, M. R. Enabling Singlet Fission by Controlling Intramolecular Charge Transfer in $\pi$-Stacked Covalent Terrylenediimide Dimers. Nat. Chem. 2016, 8, 1120-1125.

(43) Anthony, J. E. Functionalized Acenes and Heteroacenes for Organic Electronics. Chem. Rev. 2006, 106, 5028-5048.

(44) Yost, S. R.; Lee, J.; Wilson, M. W. B.; Wu, T.; McMahon, D. P.; Parkhurst, R. R.; Thompson, N. J.; Congreve, D. N.; Rao, A.; Johnson,
K.; et al. A Transferable Model for Singlet-Fission Kinetics. Nat. Chem. 2014, 6, 492-497.

(45) Petelenz, P.; Snamina, M.; Mazur, G. Charge-Transfer States in Pentacene: Dimer versus Crystal. J. Phys. Chem. C 2015, 119, 1433814342.

(46) Yamagata, H.; Norton, J.; Hontz, E.; Olivier, Y.; Beljonne, D.; Brédas, J. L.; Silbey, R. J.; Spano, F. C. The Nature of Singlet Excitons in Oligoacene Molecular Crystals. J. Chem. Phys. 2011, 134, 204703.

(47) Ito, S.; Nagami, T.; Nakano, M. Density Analysis of Intra- and Intermolecular Vibronic Couplings toward Bath Engineering for Singlet Fission. J. Phys. Chem. Lett. 2015, 6, 4972-4977.

(48) Nakano, M.; Ito, S.; Nagami, T.; Kitagawa, Y.; Kubo, T. Quantum Master Equation Approach to Singlet Fission Dynamics of Realistic/Artificial Pentacene Dimer Models: Relative Relaxation Factor Analysis. J. Phys. Chem. C 2016, 120, 22803-22815.

(49) Fujihashi, Y.; Ishizaki, A. Fluctuations in Electronic Energy Affecting Singlet Fission Dynamics and Mixing with Charge-Transfer State: Quantum Dynamics Study. J. Phys. Chem. Lett. 2016, 7, 363369.

(50) Berkelbach, T. C.; Hybertsen, M. S.; Reichman, D. R. Microscopic Theory of Singlet Exciton Fission. II. Application to Pentacene Dimers and the Role of Superexchange. J. Chem. Phys. 2013, 138, 114103.

(51) Parker, S. M.; Seideman, T.; Ratner, M. A.; Shiozaki, T. Model Hamiltonian Analysis of Singlet Fission from First Principles. J. Phys. Chem. C 2014, 118, 12700-12705.

(52) Zeng, T.; Hoffmann, R; Ananth, N. The Low-Lying Electronic States of Pentacene and Their Roles in Singlet Fission. J. Am. Chem. Soc. 2014, 136, 5755-5764.

(53) Feng, X.; Kolomeisky, A. B.; Krylov, A. I. Dissecting the Effect of Morphology on the Rates of Singlet Fission: Insights from Theory. J. Phys. Chem. C 2014, 118, 19608-19617.

(54) Teichen, P. E.; Eaves, J. D. Collective Aspects of Singlet Fission in Molecular Crystals. J. Chem. Phys. 2015, 143, 044118.

(55) Berkelbach, T. C.; Hybertsen, M. S.; Reichman, D. R. Microscopic Theory of Singlet Exciton Fission. III. Crystalline Pentacene. J. Chem. Phys. 2014, 141, 074705. 